\title{
EFICIÊNCIA DE FERTILIZANTES ORGANOMINERAIS FOSFATADOS EM MUDAS DE EUCALIPTO
}

\author{
Agronomic performance of organomineral phosphate fertilizers in eucalyptus seedlings \\ Ciro Augusto de Souza Magalhães ${ }^{1 *}$; Marina Moura Morales ${ }^{2}$; Fabiana Abreu Rezende ${ }^{1}$; Jaldes Langer ${ }^{3}$ \\ ${ }^{1}$ Pesquisador; Embrapa Agrossilvipastoril, ciro.magalhaes@embrapa.br (*autor para correspondência) e \\ fabiana.rezende@embrapa.br \\ 2 Pesquisadora; Embrapa Florestas; marina.morales@embrapa.br \\ ${ }^{3}$ Engenheiro Florestal; Flora Sinop; jaldesnp@terra.com.br
}

Artigo enviado em 08/05/2017, aceito em 01/09/2017 e publicado em 20/12/2017.

\begin{abstract}
Resumo - O uso de fertilizantes com eficiência aumentada tem crescido nos últimos anos, e o setor de produção de mudas florestais pode ser um dos beneficiados por essas tecnologias. Este estudo teve o objetivo de avaliar a eficiência de fertilizantes organominerais fosfatados sobre o desenvolvimento inicial de mudas de eucalipto. $\mathrm{O}$ experimento foi realizado em viveiro florestal localizado no município de Sinop(MT), em tubetes de $50 \mathrm{~cm}^{3}$. Foram avaliados três fertilizantes organominerais fosfatados, em um delineamento em blocos casualizados, formulados a partir da mistura e granulação, em diferentes proporções, de superfosfato triplo, fosfato natural reativo de Bayovar, conteúdo ruminal de bovinos de corte, biochar e cama-de-aviário. Essas fontes foram granuladas e aplicadas em um substrato comercial, em quatro níveis de fósforo $\left(1,4 ; 2,5 ; 3,6\right.$ e $4,7 \mathrm{mg}$ planta-1 $\left.{ }^{-1}\right)$ mais um tratamento referência. As parcelas experimentais foram compostas por 16 mudas do clone de eucalipto (Urograndis - I144), com quatro repetições. Aos 70 dias foi determinada a massa seca da parte aérea (MSPA), massa seca de raízes (MSR), teor de fósforo na parte aérea e o índice de eficiência agronômica. A produção de MSPA e MSR foi superior nas mudas com aplicação de fertilizante organomineral granulado com fosfato natural reativo de Bayovar e conteúdo ruminal. Esse fertilizante apresentou eficiência similar ao tratamento referência, podendo ser uma alternativa para a utilização na produção de mudas de eucalipto. O fertilizante organomineral granulado com superfosfato triplo, biochar e cama-deaviário não foi eficiente para o desenvolvimento inicial de mudas de eucalipto.
\end{abstract}

Palavras-chave: conteúdo ruminal, fosfato de Bayovar, biochar.

\begin{abstract}
The use of fertilizers more efficient has grown in the last years, and the seedlings production sector can be one of those benefited by these technologies. The objective of this study was to evaluate the efficiency of phosphate organomineral fertilizers on eucalyptus seedlings development. The experiment was set up in a nursery located in Sinop, state of Mato Grosso, Brazil, in $50 \mathrm{~cm}^{3}$ tubes. Three phosphate organomineral fertilizers were evaluated in a randomized block design, formulated from the mixture and granulation, in different proportions, of triple superphosphate, reactive natural phosphate from Bayovar, ruminal content of beef cattle, biochar and poultry. These sources were granulated and applied in a commercial substrate in four phosphorus levels $(1.4,2.5,3.6$ and 4.7 $\mathrm{mg}$ plant $^{-1}$ ) plus a reference treatment. The experimental plots were composed of 16 seedlings of eucalyptus clone (Urograndis - I144), with four replications. At 70 days the shoot and root dry mass, phosphorus content in shoot and the agronomic index were determined. Root and shoot production was higher in the seedling fertilized with Bayovar reactive natural phosphate granulated with ruminal content. This fertilizer could be use in seedling production. The organomineral fertilizer granulated with triple superphosphate, biochar and poultry litter was not efficient for the initial development of eucalyptus seedlings.
\end{abstract}

Keywords - ruminal content, Bayovar phosphate, biochar.

\section{INTRODUÇÃO}

O setor florestal brasileiro tem apresentado resultados crescentes, ano após ano, e em 2015 foi responsável por exportar cerca de 5,6 bilhões de dólares, somente em celulose (SNIF, 2016). O gênero Eucalyptus é o mais importante e se caracteriza por apresentar espécies de alta produtividade e com 
flexibilidade às condições edafoclimáticas brasileiras (ROCHA et al., 2013).

As áreas com histórico de alta produtividade geralmente estão relacionadas com a utilização de mudas de qualidade superior, que requerem substratos com fornecimento adequado de nutrientes durante $O$ período de viveiro. Dentre os nutrientes, o fósforo $(\mathrm{P})$ é considerado o que mais limita o desenvolvimento das mudas de eucalipto na fase inicial de produção (GRACIANO et al., 2006). Sua deficiência reduz significativamente o comprimento de raízes, acarretando em queda na qualidade das mudas (SCHAWAMBACH et al., 2005). Segundo Rocha et al. (2013), a adubação fosfatada balanceada pode tornar mais eficiente o processo de produção de mudas, reduzindo em mais de $30 \%$ as perdas, diminuindo assim o custo por unidade produzida.

Nos últimos anos, vem sendo desenvolvidos fertilizantes com tecnologias que visam aumentar sua eficiência, e as mais comuns envolvem a liberação lenta do nutriente por meio s de recobrimento dos grânulos (MACHADO; SOUZA, 2012). Já existem no mercado fertilizantes especiais, que são indicados para utilização em substratos de produção de mudas, em função de tecnologias que retardam a liberação dos nutrientes, diminuindo perdas e aumentando a eficiência. Entretanto, seu custo mais elevado dificulta a adoção por parte dos produtores de mudas florestais. Menegassi et al. (2012) comprovaram que a adubação de mudas de eucalipto com fertilizante de liberação lenta foi mais eficiente quer um formulado NPK convencional.

A união de uma matriz mineral, rica em $\mathrm{P}$, com uma matriz orgânica, os chamados fertilizantes organominerais fosfatados, tem sido alvo de inúmeras pesquisas para se obter fertilizantes mais eficientes, com custo compatível, além de minimizar a geração de resíduos orgânicos agroindustriais. Porém, a avaliação desses fertilizantes em mudas florestais é praticamente inexistente. Além disso, a solubilidade da fonte de fósforo em combinação com diferentes matrizes orgânicas, provavelmente resulta em interações distintas, e são praticamente inexistentes resultados de pesquisa com esses tipos de fertilizantes.

Diante do exposto o objetivo do trabalho foi avaliar a eficiência de fertilizantes organominerais fosfatados em mudas de eucalipto.

\section{MATERIAL E MÉTODOS}

O experimento foi conduzido no viveiro comercial de mudas da empresa Flora Sinop, localizado no município de Sinop (MT), nas coordenadas $11^{\circ} 52^{\prime} 04^{\prime \prime}$ S e $55^{\circ} 28^{\prime} 10^{\prime \prime} \mathrm{O}$ e 374 metros de altitude. O clima da região de acordo com o critério de Koppen é
Am (clima de monções) com alternância bem definida entre estação chuvosa e seca (ALVARES et al., 2013), com valores médios anuais de temperatura do ar de $25,5{ }^{\circ} \mathrm{C}$, mínima de $20,2{ }^{\circ} \mathrm{C}$ e máxima de $33,0{ }^{\circ} \mathrm{C}$; umidade relativa do ar anual de $70 \%$ e precipitação anual de $2250 \mathrm{~mm}$ (EMBRAPA, 2017), a vegetação é caracterizada como transição Cerrado - Amazônia (SOUZA et al., 2013).

O delineamento experimental utilizado foi o de blocos casualizados, em esquema fatorial $3 \times 4$, (três fertilizantes organominerais, quatro doses de P: 1,4; 2,5; 3,6 e 4,7 $\mathrm{mg}$ planta $\left.^{-1}\right)$, com três repetições de 16 plantas mais um tratamento referência. O recipiente utilizado foi o tubete cônico de $12 \mathrm{~cm}$ de comprimento e 50 $\mathrm{cm}^{3}$. Os tubetes foram previamente esterilizados em água a $80^{\circ} \mathrm{C}$, por 30 segundos.

O tratamento referência é caracterizado pelo fornecimento de $\mathrm{P}$ via termofosfato magnesiano (14\% de $\mathrm{P}_{2} \mathrm{O}_{5}$ solúvel em ácido cítrico - $2 \mathrm{~kg} \mathrm{~m}^{-3}$, ou 2,2 $\mathrm{mg}$ de $\mathrm{P}$ planta $\left.^{-1}\right)$ e BASACOT MINI 6M® $(13,06,13,02$ e $10 \%$ de $\mathrm{N}, \mathrm{P}_{2} \mathrm{O}_{5}, \mathrm{~K}_{2} \mathrm{O}, \mathrm{MgO}$ e $\mathrm{S}$, respectivamente, além de 0,26; 0,2; 0,2; 0,05; 0,05; 0,06 e 0,015\% de Fe, $\mathrm{B}, \mathrm{Zn}, \mathrm{Cu}, \mathrm{Mn}$ e Mo, respectivamente) $-3 \mathrm{~kg} \mathrm{~m}^{-3}$, ou 1,4 mg de P planta ${ }^{-1}$.

Os fertilizantes organominerais foram obtidos a partir da moagem e peneiramento (malha de $250 \mu \mathrm{m}$ ) de fontes orgânicas (conteúdo ruminal de bovinos de corte, cama-de-aviário e biocarvão de serragem de madeira) e minerais (superfosfato triplo - 46\% de $\mathrm{P}_{2} \mathrm{O}_{5}$ total e fosfato natural reativo de Bayovar - 34\% de $\mathrm{P}_{2} \mathrm{O}_{5}$ total), homogeneização prévia em misturador e granulação em disco granulador, com adição de silicato de sódio $(0,4 \% \mathrm{p} / \mathrm{p})$ via aspersão e bentonita $(2,4 \%$ $\mathrm{p} / \mathrm{p})$, Quadro 1.

As mudas de eucalipto foram obtidas do clone comercial (I144), híbrido de Eucalyptus urophylla x Eucalyptus grandis, produzidas a partir da técnica de miniestaquia (XAVIER et al., 2009). O substrato utilizado no experimento foi composto de fibra de coco e casca de arroz carbonizada, na proporção de 1:1 (v:v). Após o estaqueamento, os tubetes receberam uma fina camada de vermiculita expandida, e permaneceram em casa-de-vegetação por 30 dias, com irrigações periódicas por microaspersão, de 30 em 30 minutos, durante todo o dia. Após esse período, as mudas foram transferidas para área com insolação total, recebendo irrigações diárias de $10 \mathrm{~mm}$, divididas em 5 irrigações. 
Quadro 1. Fertilizantes organominerais produzidos e avaliados no experimento.

\begin{tabular}{|c|c|c|}
\hline Fórmula & Composição ( $\%$ p/p) & $\begin{array}{c}\text { Teor de } \\
\mathbf{P}_{2} \mathrm{O}_{5} \text { total } \\
\left(\mathrm{g} \mathrm{kg}^{-1}\right)\end{array}$ \\
\hline STCR & $\begin{array}{l}\text { Superfosfato triplo } \\
(40 \%)+\text { conteúdo } \\
\text { ruminal de bovinos } \\
(57,2 \%)\end{array}$ & 190 \\
\hline BAYCR & $\begin{array}{c}\text { Fosfato natural reativo } \\
\text { de Bayovar }(60 \%)+ \\
\text { conteúdo ruminal de } \\
\text { bovinos }(37,2 \%)\end{array}$ & 208 \\
\hline STBIOCA & $\begin{array}{l}\text { Superfosfato triplo } \\
(40 \%)+\text { biochar } \\
(27,2 \%)+\text { cama-de- } \\
\text { aviário }(30 \%)\end{array}$ & 187 \\
\hline
\end{tabular}

Aos 70 dias, final do período de avaliação, foi realizada a contagem das mudas vivas, e após as mudas foram retiradas dos tubetes e separadas a parte aérea das raízes, esta última com lavagem para remoção do substrato residual. Ambas as frações foram secas em estufa de circulação forçada a $65{ }^{\circ} \mathrm{C}$ até massa constante, para a obtenção da produção de massa seca da parte aérea (MSPA) e massa seca das raízes (MSR). Foi calculada a produção média por planta, dividindose a massa seca total pelo número de plantas de cada parcela. Em seguida, a MSPA foi triturada em moinho de facas $(1 \mathrm{~mm})$ para a quantificação do teor de P total, via digestão seca seguida de determinação por espectofotometria com azul-de- molibdênio e leitura a $660 \mathrm{~nm}$, de acordo com Embrapa (2009).

A partir do teor de $\mathrm{P}$ total na parte aérea e da sua massa seca, determinou-se o $\mathrm{P}$ acumulado na MSPA, por:

$$
A c=\frac{M S P A \times[P]}{1000}
$$

Em que: Ac = acúmulo de fósforo $(\mathrm{P}), \mathrm{mg}$ planta $^{-1}$; MSPA = massa seca da parte aérea, $\left.\operatorname{mg~planta}^{-1} ; \mathrm{P}\right]=$ teor de $\mathrm{P}$ na parte aérea, $\mathrm{g} \mathrm{kg}^{-1}$.

A eficiência dos fertilizantes quanto à produção de matéria seca foi obtido por:

$$
I E A=\left[\frac{\left(Y_{2}-Y_{1}\right)}{\left(Y_{a}-Y_{1}\right)}\right] \times 100
$$

Em que: IEA (\%) = Índice de eficiência agronômica; $Y_{1}=$ MSPA obtida na dose 0 de fósforo (sem adição de fertilizante organomineral - média de 9 parcelas experimentais); $Y_{2}=$ MSPA obtida com a fonte na dose $\mathrm{n}\left(2,5 ; 3,6\right.$ e 4,7 $\mathrm{mg} \mathrm{dm}^{-3}$ de P); e $Y_{3}=$ MSPA obtida pela tratamento referência.

A avaliação da eficiência agronômica das doses dos fertilizantes fosfatados e a interação entre eles foram obtidos por análise de variância. Para avaliação do crescimento das mudas (MSPA, MSR e matéria seca total) e a eficiência nutricional (teor de P na MSPA, P acumulado), foram ajustadas equações de regressão, com uso do programa estatístico SISVAR versão 5.3 (FERREIRA, 2011).

\section{RESULTADOS E DISCUSSÃO}

O rendimento de MSPA, para os três fertilizantes, apresentou comportamento quadrático em função das doses de $\mathrm{P}$ dos fertilizantes (Figura 1). Houve interação significativa $(p<0,001)$ entre fertilizantes e doses. A produção de MSPA do tratamento referência foi, em média de $613 \mathrm{mg}$ planta $^{-1}$. O fertilizante BAYCR, a partir da dose $3,6 \mathrm{mg} \mathrm{dm}^{-3}$ de $P$ planta ${ }^{-1}$ proporcionou produção semelhante ao tratamento referência. A produção máxima foi obtida pelo fertilizante BAYCR, de $654 \mathrm{mg}$ planta $^{-1}$, na dose de 4,7 $\mathrm{mg}$ de $\mathrm{P}$ planta $^{-1}$. O STCR respondeu ao aumento da dose de $\mathrm{P}$, mas foi inferior ao tratamento referência mesmo na maior dose. Já o fertilizante STBIOCA não respondeu ao aumento das doses de $\mathrm{P}$ via fertilizante organomineral.

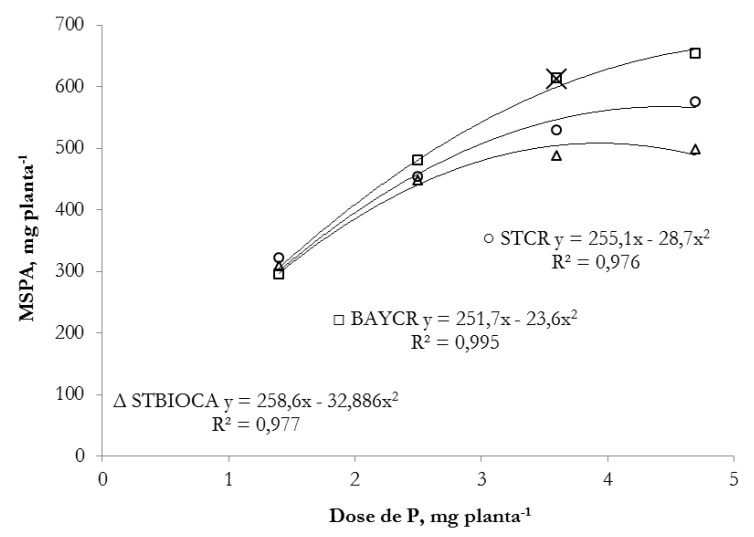

Figura 1. Produção de massa seca da parte aérea (MSPA) de Eucaliptus Urograndis - clone I144, em função de doses de fertilizantes organominerais fosfatados (X representa o valor obtido no tratamento referência; STCR: superfosfato triplo + conteúdo ruminal; BAYCR: fosfato de Bayovar + conteúdo ruminal; STBIOCA: superfosfato triplo + biochar + cama-de-aviário). 
O efeito das doses de $\mathrm{P}$ no crescimento radicular também variou com os fertilizantes (Figura 2) e o comportamento foi muito similar ao ocorrido para a produção de MSPA. A produção máxima foi obtida pelo fertilizante BAYCR, de $270 \mathrm{mg}$ planta $^{-1}$, na dose de 4,7 mg de P planta ${ }^{-1}$. Para o fertilizante STCR na maior dose de $\mathrm{P}$ sua produção de raízes foi semelhante ao tratamento referência. A produção de massa de raízes tem estreita relação com a sobrevivência das mesmas após o plantio, pois mudas com sistema radicular mais desenvolvido resistem mais ao estresse do plantio, além de ter maior capacidade de absorver água e nutrientes (MAFIA et al., 2005).

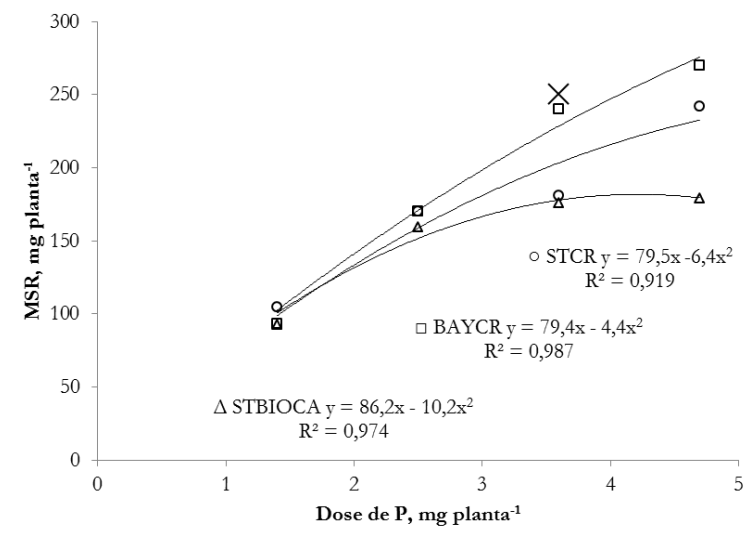

Figura 2. Produção de massa seca radicular (MSR) de Eucaliptus Urograndis - clone I144, em função de doses de fertilizantes organominerais fosfatados ( $\mathrm{X}$ representa o valor obtido no tratamento referência).

A produção de massa seca total seguiu o comportamento quadrático (Figura 3), e a máxima produção foi a do fertilizante BAYCR, de $924 \mathrm{mg}$ planta $^{-1}$, na dose de 4,7 mg de P planta ${ }^{-1}$. Rocha et al. (2013), também avaliando a produção de mudas de eucalipto em função de doses de $\mathrm{P}$, via superfosfato simples $(0,0 ; 1,3 ; 2,6$ e 5,2 $\mathrm{mg}$ de $\mathrm{P}$ por planta), determinaram que o fornecimento de $3,6 \mathrm{mg}$ de $\mathrm{P}$ planta $^{-1}$ proporcionou a produção máxima de massa seca da parte aérea $\left(1160 \mathrm{mg}\right.$ planta $^{-1}$ aos 90 dias de avaliação) e que doses superiores a $4 \mathrm{mg}$ planta $^{-1}$ prejudicaram o desenvolvimento e a qualidade das mudas.

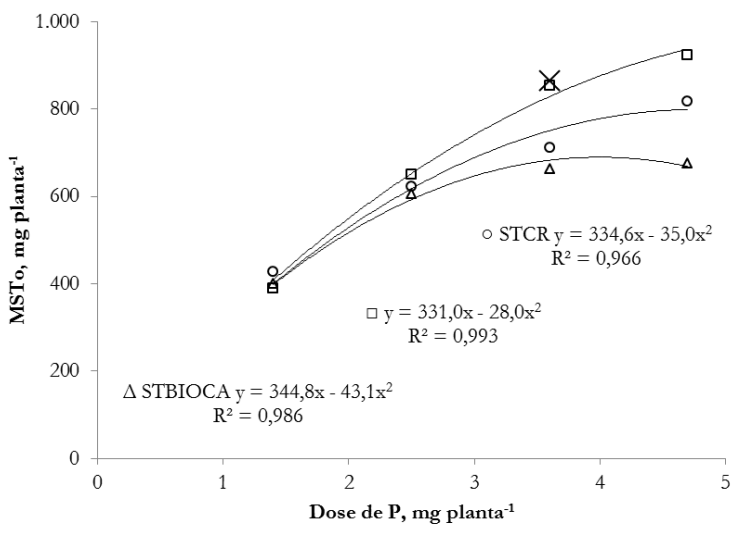

Figura 3. Produção de massa seca total (MSTo) de Eucaliptus Urograndis - clone I144, em função de doses de fertilizante organominerais fosfatados (X representa o valor obtido no tratamento referência).

Para todas as estimativas de produção de massa seca (parte aérea, raízes e total), para o fertilizante BAYCR, que apresentou os maiores valores, não foi possível determinar a produção máxima, já que as doses estimadas seriam superiores a dose máxima avaliada. Isso indica que as mudas de eucalipto têm potencial para responder em produção de massa seca para doses superiores a 4,7 mg de $\mathrm{P}$ planta $^{-1}$, diferentemente do encontrado por Rocha et al. (2013).

$\mathrm{O}$ teor de $\mathrm{P}$ na MSPA sofreu pouca influência das doses de fertilizantes, e foi menor para o BAYCR na maior dose $\left(4,7 \mathrm{mg}\right.$ de $\mathrm{P}$ planta $\left.^{-1}\right)$, possivelmente devido a um efeito de diluição, já que nessa dose a produção de MSPA foi a maior observada.

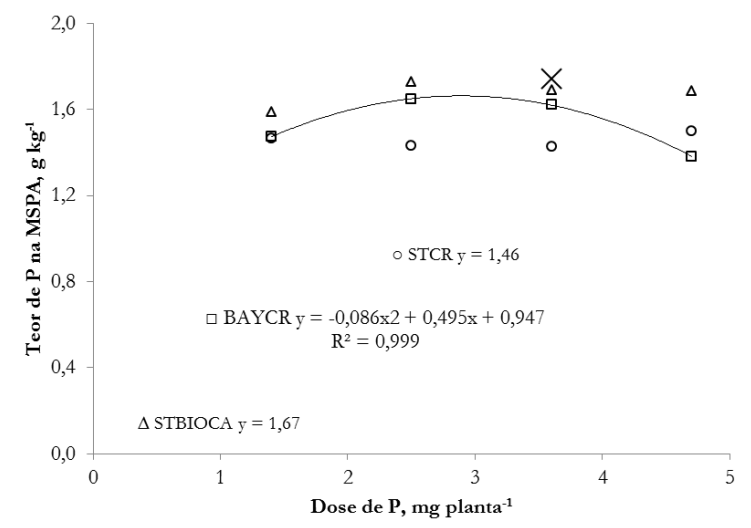

Figura 4. Teor de fósforo na massa seca da parte aérea de mudas de Eucaliptus Urograndis - clone I144, em função de doses de fertilizantes organominerais fosfatados (X representa o valor obtido no tratamento referência). 
Para o acúmulo de $\mathrm{P}$ na MSPA, houve diferenças entre os fertilizantes (Figura 5), sendo que BAYCR apresentou acúmulo maior, de 0,968 mg planta $^{-1}$ de $\mathrm{P}$, na dose 4,4 mg planta ${ }^{-1}$.

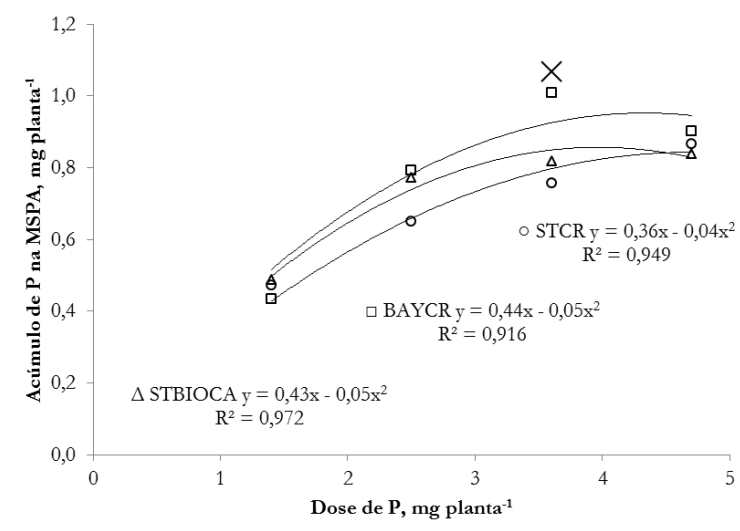

Figura 5. Acúmulo de fósforo na massa seca da parte aérea de mudas de Eucaliptus Urograndis - clone I144, em função de doses de fertilizantes organominerais fosfatados (X representa o valor obtido no tratamento referência).

O índice de eficiência agronômica (Tabela 2) demonstra a superioridade do fertilizante BAYCR, em comparação aos outros dois fertilizantes organominerais na produção de mudas de eucalipto clone I144. A partir da dose 3,6 $\mathrm{mg}$ planta $^{-1}$ seu valor se igualou ao tratamento referência. Para o STCR o IEA se elevou com o aumento da dose, porém não se igualou ao tratamento referência, enquanto $O$ STBIOCA não superou $62 \%$ de IEA, comprovando sua menor eficiência para a produção de MSPA das mudas de eucalipto.

Tabela 2. Índice de eficiência agronômica (\%) dos fertilizantes organominerais fosfatados em mudas de Eucaliptus Urograndis - clone I144.

\begin{tabular}{ccccc}
\hline \multirow{2}{*}{ Fertilizante } & \multicolumn{3}{c}{ Dose de $\mathrm{P}\left(\mathrm{mg}\right.$ planta $\left.^{-1}\right)$} & \multirow{2}{*}{ Média } \\
\cline { 2 - 4 } & 2,5 & 3,6 & 4,7 & \\
\hline STCR & $48 \mathrm{Ab}$ & $73 \mathrm{Bb}$ & $88 \mathrm{Ba}$ & 69 \\
BAYCR & $56 \mathrm{Ab}$ & $100 \mathrm{Aa}$ & $113 \mathrm{Aa}$ & 90 \\
STBIOCA & $45 \mathrm{Aa}$ & $58 \mathrm{Ba}$ & $62 \mathrm{Ca}$ & 55 \\
\hline
\end{tabular}

*Letras iguais, maiúsculas nas colunas; minúsculas nas linhas, não diferem entre si, pelo teste de Scott-Knott, a 5\% de probabilidade.

Rezende et al. (2016) avaliaram a adição de 2 tipos de biochar em substrato para produção de mudas de teca (Tectona grandis) e definiram que $25 \%$ de biochar ativado na composição do substrato foi suficiente para aumentar o crescimento das mudas. Porém, no presente estudo, a combinação superfosfato triplo + biochar + cama-de-aviário não resultou em ganho de produção de MSPA das mudas submetidas a essa fórmula. Aspectos físicos e químicos dessa formulação precisam ser melhor estudados a fim de elucidar os mecanismos envolvidos entre essas fontes que não possibilitaram o aproveitamento do $\mathrm{P}$ presente nela.

\section{CONCLUSÕES}

1. O fertilizante organomineral granulado com fosfato natural reativo de Bayovar e conteúdo ruminal de bovinos de corte propiciou desenvolvimento inicial de mudas de eucalipto urograndis- clone I144, similar ao tratamento referência, podendo ser uma alternativa para a utilização na produção de mudas de eucalipto.

2. O fertilizante organomineral granulado com superfosfato triplo, biochar e cama-de-aviário não foi eficiente para o desenvolvimento inicial de mudas de eucalipto.

\section{AGRADECIMENTOS}

À FAPEMAT (Fundação de Amparo à Pesquisa do Estado de Mato Grosso), pelo apoio financeiro (projeto aprovado no Edital Universal 2014 - processo 163535/2014) e à Flora Sinop, pelo apoio na condução do ensaio.

\section{REFERÊNCIAS}

ALVARES C.A., STAPE J.L., SENTELHAS P.C., DE MORAES G., LEONARDO J., SPAROVEK G., 2013. Köppen's climate classification map for Brazil. Meteorologische Zeitschrift, v. 22, p. 711-728, 2013.

EMBRAPA. Manual de de análises químicas de solos, plantas e fertilizantes. Brasília, DF: EMBRAPA Informação Tecnológica, 2009. 627 p.

EMBRAPA. Estação meteorológica automática. Disponível em https:/ /www.embrapabr/agrossilvipastoril/estacaometeorologica [Verificado em Abr. 2017].

FERREIRA, Daniel Furtado. Sisvar: a computer statistical analysis system. Ciência \& Agrotecnologia, v. 35, n. 6, p. 1039-1042, Dec. 2011. http:/ /dx.doi.org/10.1590/S141370542011000600001 .

GRACIANO, C.; GOYA, J. F.; FRANGI, J. L.; GUIAMENT, J. J. Fertilization with phosphorus increases soil nitrogen absorption in young plants of Eucalyptus grandis. Forest Ecology and Management, v. 236, p. 202-210, 2006. 
MACHADO, V. J.; SOUZA, C. H. E. Disponibilidade de fósforo em solos com diferentes texturas após aplicação de doses crescentes de fosfato monoamônico de liberação lenta. Bioscience Journal, v. 28, p.1-7, 2012.

MENEGASSI, A. D.; SILVEIRA, E. R.; FERRONATO, M. L.; REINER, D. A. Produção de mudas de eucalipto sob diferentes fontes de adubação. $4^{\circ}$ Congresso Florestal Paranaense. 2012. Disponível em http://malinovski.com.br/CongressoFlorestal/Trabalh os/05-Silvicultura/SIL-Artigo-26.pdf [Verificado em Abr. 2017].

MAFIA, R. G.; ALFENAS, A. C.; SIQUEIRA, L.; FERREIRA, E. M.; LEITE, H. G.; CAVALLAZZI, J. R. P. Critério técnico para determinação da idade ótima de mudas de eucalipto para plantio. Revista Arvore, v. 29, p. 947-953, 2005.

REZENDE, F. A.; SANTOS, V. A. H. F.; MAIA, C. M. B. F.; MORALES, M. M. Biochar in substrate composition for production of teak seedlings. Pesquisa Agropecuária Brasileira, v. 51, p. 449-1456, 2016. https://dx.doi.org/10.1590/s0100-

$204 \times 2016000900043$.
ROCHA, J. H. T.; PIETRO, M. R.; BORELLI, K.; BACKES, C.; NEVES, M. B. Produção e desenvolvimento de mudas de eucalipto em função de doses de fósforo. Cerne, v. 19, p. 535-543, 2013.

SCHAWAMBACH, J.; FADANELLI, C.; FET'T NETO, A. G. Mineral nutrition and adventitious rooting in microcuttings of Eucalyptus globules. Tree Physiology, v. 25, p. 487-494, 2005.

SNIF - SISTEMA NACIONAL DE INFORMAÇÕES FLORESTAIS. Produção florestal. Serviço Florestal Brasileiro, 2016. Disponível em https://

http://www.florestal.gov.br/snif/images/stories/Prod ucaoFlorestal/boletim_snif_producao $\% 20$ florestal_dez 2016_final.pdf

SOUZA, A. P.; MOTA, L. L.; ZAMADEI, T.; MARTIM, C. C.; ALMEIDA, F. T.; PAULINO, J. Classificação climática e balanço hídrico climatológico no estado de Mato Grosso. Nativa, v. 01, p. 34-43, out./dez., 2013.

XAVIER, A.; WENDLING, I.; SILVA, R. L. Silvicultura clonal: princípios e técnicas. Viçosa, MG: Ed. UFV. 272p. 2009. 